\title{
Power Angle Small-Signal Stability Analysis of Grid-Forming Wind Turbine Inverter Based on VSM Control
}

\author{
Liang $\mathrm{Lu}$ \\ Department of Wind Energy \\ Technical University of Denmark \\ Roskilde, Denmark \\ lilu@dtu.dk
}

\author{
Ömer Göksu \\ Department of Wind Energy \\ Technical University of Denmark \\ Roskilde, Denmark \\ omeg@dtu.dk
}

\author{
Nicolaos A. Cutululis \\ Department of Wind Energy \\ Technical University of Denmark \\ Roskilde, Denmark \\ niac@dtu.dk
}

\begin{abstract}
This paper introduces a virtual synchronous machine (VSM) control scheme which is based on a minimized emulation of the model of a synchronous generator (SG) for a grid-forming wind turbine (WT) inverter. Based on this VSM control scheme, state-space representation and block diagram of the small-signal model for the VSM-controlled grid-forming inverter connected to an infinite bus are derived, which are used for small-signal stability analysis of the power angle in the VSM control scheme. This power angle small-signal stability (PAS) analysis is proposed as a simpler way for analyzing the influence on the rotor angle stability of power system from large share of VSM-controlled grid-forming inverters. Influence of parameters in the power loop of the control scheme on the PAS is investigated by analyzing the eigenvalue trajectories of the state matrix.
\end{abstract}

Keywords-grid-forming WT inverter, virtual synchronous machine (VSM) control, rotor angle stability, power angle smallsignal stability (PAS), eigenvalue trajectory

\section{INTRODUCTION}

Synchronous generators (SGs) are the main power sources in our traditional power system today. Due to the coupling of their mechanical rotating speed and the frequency of the power grid, their huge inertia makes it difficult to have a large rate of change of frequency (RoCoF) when there is a large power imbalance between generation and load (resulting in a frequency event). Furthermore, their power input can be regulated automatically to counteract the power imbalance, which stabilizes frequency back to a steady state. These two aspects help to maintain the frequency stability of the power system.

However, for state of the art wind turbines (WTs), namely type-3 WTs or type-4 WTs, either with optimal speed control for maximum power point tracking (MPPT) or with back-to-back converters fully interfaced with the power grid, make their mechanical rotating speed decoupled from the frequency of the grid. As a result, the inertia of their rotating masses will not help to limit the RoCoF, as an SG does, when there is a frequency event. Besides, another significant difference between a WT and an SG is that the power source for a WT is not controllable, which makes it difficult for a WT to change its power output as demanded. These two differences bring a big challenge for the frequency stability of our power system with more and more wind power being integrated into our power grid nowadays.

This work has received funding from the European Union's Horizon 2020 research and innovation program under agreement No. 727680 (TotalControl)

This work has also received funding from project PowerKey (EUDP Project No. 12558)
To avoid this problem, a straightforward idea has been controlling a WT to behave in the same or similar way as an SG. This is how the concept of virtual synchronous machine (VSM) control comes from [1]-[4]. Specifically, VSM control is one category of control schemes applied to converters, which enables WTs to perform similar frequency control as SGs, by adding a SG model in the control schemes.

Considering the big differences between a converter and an SG in structures and characteristics, it is feasible only to emulate the desirable properties of an SG rather than also to include the undesirable ones, like complex electromagnetic dynamics. In this way, the control scheme will also be simpler, which helps to keep the advantages of converter control, like fast response and flexibility. Hence, it is considered as an objective in this study to get a minimized emulation of the SG model for the VSM control scheme, to simplify the control scheme as much as possible while keeping qualified frequency control capability from the WT. According to this motivation, a simplified VSM control scheme is proposed [5], which is also specially designed for WT applications.

As stability analysis is very important for a control system, it is essential to analyze the stability and design the parameters of our proposed VSM control scheme, which is the main topic of this paper. To get started, this paper focuses on the small-signal stability of the power angle in the power loop of the VSM control scheme. Especially, it is another goal in this paper to have a simple and practical analysis with focus on the outer power loop only, considering the different bandwidths of inner and outer loops. Moreover, state space representation and eigenvalue sensitivity analysis are used for easiness of parameter design.

On this topic, there has been relevant research. There is small-signal stability analysis carried out using transfer functions and Bode diagrams [6]-[10]. There are publications mentioning small-signal stability but with other focuses, like synchronous frequency resonance (SFR) [6], power oscillation and suppression [7]; and some of them do not include power angle [10]-[12] or parameter design [12][13]. There are some papers most relevant with smallsignal stability analysis including power angle as a state variable [13]-[24]. However, a detailed state space modeling of the whole converter control scheme and electric circuits is used in these papers, some with more than ten state variables, which is opposite to the goal of simplification in this paper. Indeed, there are some simpler representations [25][26], but they do not focus on the small-signal stability analysis and parameter design of the VSM-controlled inverter itself. Last but not the least, all the previous studies are based on VSM 
control schemes specific to those studies, like synchronverter [10], [12], [16]-[19], schemes developed by SINTEF [20][24], Osaka University [11], Kawasaki Technology Co. [24] and others [25]. Therefore, it is also essential to analyze the small-signal stability of the proposed VSM control scheme, especially with the focus and goal mentioned above, which has not been thoroughly investigated before in the literature.

The rest of paper is organized as follows. Section II gives an introduction of the proposed VSM control scheme, of which small-signal stability analysis of the power angle and parameter design is illustrated in detail in Section III, before concluding in Section IV.

\section{PROPOSED VSM CONTROL SCHEME}

The motivation to propose a different VSM control scheme from those in the literature considers three aspects. Firstly, this scheme is specially designed for WTs, where type-4 WT is focused in our research. That is to say, special structure of WTs and specific requests from grid codes for wind power need to be considered and are adopted in the scheme. Secondly, this scheme is applied only for equipping WTs with frequency control capability, namely frequency containment reserve (FCR) [27]. Hence, voltage control capability is not required and optimized in the design of the scheme. Thirdly, this scheme is designed to give a minimized emulation of the SG model, which is supposed to guarantee qualified frequency control capability for WTs and meanwhile reduce undesired characteristics of SGs as much as possible.

The main blocks of the proposed control scheme are introduced briefly as follows.

\section{A. Swing Equation}

The equation representing the mechanical motion of an SG [28] is

$$
\frac{2 H}{\omega_{n}} \frac{d^{2} \delta}{d t^{2}}=T_{m}-T_{e}-\frac{D}{\omega_{n}} \frac{d \delta}{d t}
$$

where

$H \quad=$ inertia constant in $M W \cdot s / M V A$

$\omega_{n} \quad=$ rated angular velocity in electrical $\mathrm{rad} / \mathrm{s}$

$\delta \quad=$ angular position in electrical radians

$t \quad=$ time in $s$

$T_{m} \quad=$ mechanical torque in $\mathrm{pu}$

$T_{e} \quad=$ electromagnetic torque in pu

$D$ = damping factor in pu torque/pu speed deviation

It is referred to as the swing equation as it represents swings in rotor angle $\delta$ during disturbances. For analysis for its dynamics, (1) is expressed in the state-space form, which consists of two first-order differential equations [28], becoming

$$
\begin{gathered}
\frac{d \Delta \omega_{r}}{d t}=\frac{1}{2 H}\left(T_{m}-T_{e}-D \Delta \omega_{r}\right) \\
\frac{d \delta}{d t}=\omega_{n} \Delta \omega_{r}
\end{gathered}
$$

where

$\omega_{r} \quad=$ angular velocity in electrical $\mathrm{rad} / \mathrm{s}$

$\Delta \omega_{r}=$ deviation of angular velocity in pu

$$
=\left(\omega_{r}-\omega_{n}\right) / \omega_{n}
$$

From (2) it is easy to find that a big inertia (reflected by the value of $H$ ) helps to limit the value of $d \Delta \omega_{r} / d t$, which is equivalent to RoCoF. This is how an SG helps to keep the frequency stability by avoiding a large RoCoF, and this is the very reason that a VSM control scheme needs to include the swing equation to emulate the inertial response. A swing equation branch is based on (2) and (3) in the proposed VSM control scheme.

\section{B. Governor}

The governor for an SG with speed-droop characteristic is characterized as a proportional controller with a gain of $1 / R$, shown in Fig. 1 below [28].

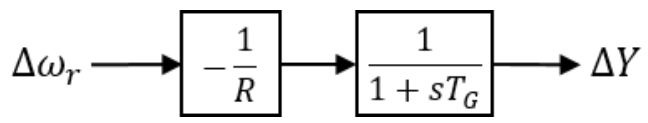

Fig. 1. Block diagram of a speed governor with droop

The parameter $R$, referred to as speed regulation or droop, determines the steady-state speed versus load characteristic of the SG. It is equal to the ratio of speed deviation $\left(\Delta \omega_{r}\right)$ or frequency deviation $(\Delta f)$ to change in valve/gate position $(\Delta Y)$ or power output $(\Delta P)$. It is the governor that regulates the power output of SG according to the change of grid frequency and supports the grid to bring the frequency back to a new steady-state value during the FCR process.

Inspired by this, a governor branch is included in the proposed VSM control scheme, of which a droop coefficient enables the WT to have the same frequency control capability as an SG does. More importantly, similar to the right block in Fig. 1, a time delay block is also included in the governor branch of the proposed VSM control scheme, to represent the response time of different components in frequency control scheme of the WT, like pitch control, speed control or energy storage. This makes the proposed VSM control scheme specially designed for WT applications.

\section{Reference Voltage Vector}

The proposed VSM control scheme consists of three cascaded control loops: power loop, voltage loop and current loop. Power loop is the outermost one, voltage loop in the middle, and current loop is innermost.

The power loop generates a reference voltage vector to the voltage loop, which consists of two components: magnitude reference and phase angle reference. The phase angle reference is the power angle $\delta$ calculated after the swing equation branch. Considering the research question of frequency control and goal of qualified simplicity in this work, the magnitude reference is simply set to the reference value of voltage at point of common coupling (PCC).

\section{Inner Control Loops}

For the inner voltage control loop and current control loop, proportional-resonant (PR) controllers are used considering their better transient performance, limited total harmonics distortion (THD) and ease of implementation without $d q$ transformation, compared with proportionalintegral (PI) controllers [29].

In summary, the block diagram of the proposed VSM control scheme is shown in Fig. 2 below. In the governor 
branch, $D_{r}$ is the droop coefficient and $T_{g}$ represents the response time of frequency control capability from WTs.

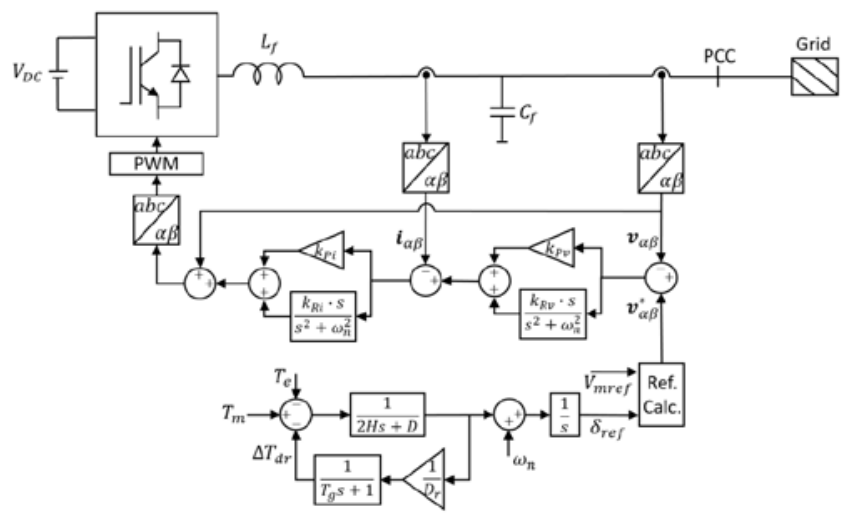

Fig. 2 The proposed VSM control scheme

\section{Power ANGLE SMALL-SignAl StABILITY}

A grid-forming inverter [30] controlled by a VSM control scheme performs similarly as an SG in frequency and voltage control capabilities. As there is a virtual power angle used in the VSM control scheme, the concepts of smallsignal stability and transient stability within the scope of rotor angle stability for an SG therefore can be transplanted to VSM-controlled grid-forming inverters. This work builds the power angle small-signal stability (PAS) method.

Considering the different bandwidths of the power loop and inner control loops, the latter are considered ideal (i.e. regarded as a unity gain with ideal reference tracking) when the PAS is investigated. In this way, the analysis of PAS is somehow simplified and parametric design can be focused only on the parameters in the power loop, which is an important procedure in the design of the proposed VSM control scheme.

\section{A. A Single-Machine Infinite Bus System}

For better understanding basic concepts and gaining experience with analytical methods, the PAS is studied of a single VSM-controlled inverter connected to an infinite bus through transmission lines. A general system configuration is shown in Fig. 3 below.

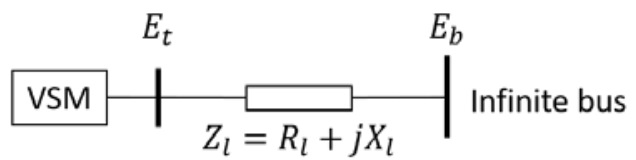

Fig. 3 A single-VSM infinite bus system

With the inverter control represented by the power loop only and all resistances neglected, the system representation is as shown in Fig. 4 below.

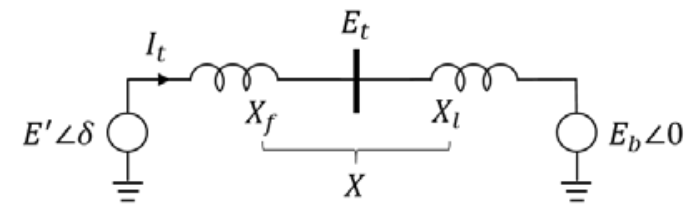

Fig. 4 A reduced system representation

Here $E^{\prime}$ is the virtual electromotive force (EMF) behind the filter reactance $X_{f}$. Choosing $E_{b}$ as a synchronously rotating reference phasor, the power angle $\delta$ is measured as the angle by which $E^{\prime}$ leads $E_{b}$.

$$
\mathbf{I}_{t}=\frac{E^{\prime} \angle \delta-E_{b} \angle 0}{j X}=\frac{E^{\prime}(\cos \delta+j \sin \delta)-E_{b}}{j X}
$$

The complex power behind $X_{f}$ is given by

$$
\begin{aligned}
S & =P+j Q=\mathbf{E}^{\prime} \mathbf{I}_{t}^{*} \\
& =\frac{E^{\prime} E_{b} \sin \delta}{X}+j \frac{E^{\prime}\left(E^{\prime}-E_{b} \cos \delta\right)}{X}
\end{aligned}
$$

\section{B. State-Space Representation}

As shown in Fig. 2 we can obtain

$$
\begin{gathered}
\frac{d \Delta \omega}{d t}=\frac{1}{2 H}\left(T_{m}-T_{e}-D \Delta \omega-\Delta T_{d r}\right) \\
\frac{d \delta}{d t}=\omega_{n} \Delta \omega \\
\Delta T_{d r}=\frac{1}{T_{g} s+1} \frac{1}{D_{r}} \cdot \Delta \omega
\end{gathered}
$$

where $\Delta T_{d r}$ is the additional torque in per unit generated for frequency control. Except that $\delta$ is in electrical radians and $\omega_{n}$ is in radians per second, all the other variables are in per unit.

In per unit, the electromagnetic torque $T_{e}$ is equal to the electromagnetic power $P$ in (5). Hence

$$
T_{e}=P=\frac{E^{\prime} E_{b} \sin \delta}{X}
$$

Linearizing (9) at an initial operating point $\delta=\delta_{0}$ yields

$$
\Delta T_{e}=\frac{\partial T_{e}}{\partial \delta} \Delta \delta=\frac{E^{\prime} E_{b}}{X} \cos \delta_{0} \cdot \Delta \delta
$$

Linearizing (6) and substituting for $\Delta T_{e}$ in (10) yields

$$
\frac{d \Delta \omega}{d t}=\frac{1}{2 H}\left(\Delta T_{m}-K_{s} \Delta \delta-D \Delta \omega-\Delta T_{d r}\right)
$$

where $K_{s}$ is the synchronizing torque coefficient in per unit torque/radians given by

$$
K_{s}=\frac{E^{\prime} E_{b}}{X} \cos \delta_{0}
$$

Linearizing (7), rate of change of power angle is obtained as

$$
\frac{d \Delta \delta}{d t}=\omega_{n} \Delta \omega
$$

Rewriting (8),

$$
\frac{d \Delta T_{d r}}{d t}=-\frac{1}{T_{g}} \Delta T_{d r}+\frac{1}{T_{g} \cdot D_{r}} \Delta \omega
$$

Writing (11), (13) and (14) in the vector-matrix form, (15) is obtained

$$
\frac{d}{d t}\left[\begin{array}{c}
\Delta \omega \\
\Delta \delta \\
\Delta T_{d r}
\end{array}\right]=\left[\begin{array}{ccc}
-\frac{D}{2 H} & -\frac{K_{s}}{2 H} & -\frac{1}{2 H} \\
\omega_{n} & 0 & 0 \\
\frac{1}{T_{g} D_{r}} & 0 & -\frac{1}{T_{g}}
\end{array}\right]\left[\begin{array}{c}
\Delta \omega \\
\Delta \delta \\
\Delta T_{d r}
\end{array}\right]+\left[\begin{array}{c}
\frac{1}{2 H} \\
0 \\
0
\end{array}\right] \Delta T_{m}
$$


This is of the form $\dot{\mathbf{x}}=\mathbf{A x}+\mathbf{b} u$. The elements of state matrix $\mathbf{A}$ are seen to be dependent on system parameters $X$, $H, D, D_{r}, T_{g}$, and initial operation condition represented by $E^{\prime}$ and $\delta_{0}$. The block diagram representation is shown in Fig. 5.

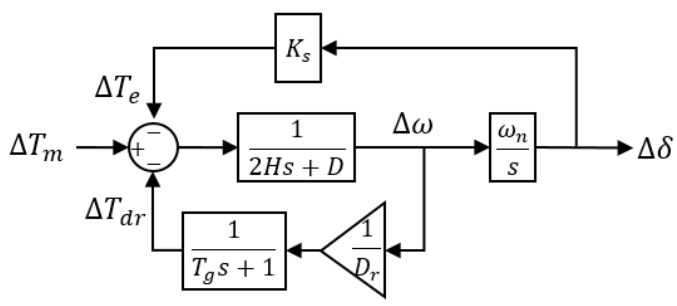

Fig. 5 Block diagram of a single-VSM infinite bus system

\section{Verification}

To test the correctness of the derived PAS model, differences of time-domain simulation results between the PAS model and a detailed model, which consists of a VSMcontrolled inverter with PWM switching connected to an infinite bus are compared. Both models are built in MATLAB Simulink.

The PAS model is built as the block diagram shown in Fig. 5, with the parameter values in TABLE I.

The circuit of the detailed model is shown in Fig. 2, in which the grid is modeled by a voltage source. In this model, power is used as input instead of torque. The values of parameters are shown in TABLE II.

In the simulations, at $t=1 \mathrm{~s}$ the mechanical torque is dropped by $0.5 \mathrm{pu}$ or mechanical power by $5000 \mathrm{~W}$, and the response of $\Delta \omega$ is recorded and compared. The results are shown in Fig. 6.

From the comparison, we can see these two models match very well in the first drop and recovery process of the angular speed. The only big difference is that the detailed model has a bigger overshoot, but this overshoot is relatively small compared with the nadir of the whole process. Considering that the inner voltage and current control loops and PWM of the inverter have been neglected, this PAS model gives a satisfying representation of the whole system.

Therefore, this PAS model provides a simpler way for focusing on the power loop analysis and PAS of the inverter. This will be especially helpful for analyzing the rotor angle stability of the power system in the future when large share of grid-forming inverters are integrated to the power system.
TABLE I. PARAMETERS OF THE PAS MODEL

\begin{tabular}{ll}
\hline Parameter & Value \\
\hline Synchronizing torque coefficient, $K_{s}$ & 4.3522 \\
Rated electrical angular speed, $\omega_{n}$ & $314 \mathrm{rad} / \mathrm{s}$ \\
Inertia constant, $H$ & $0.1775 \mathrm{~W} \cdot \mathrm{s} / \mathrm{VA}$ \\
Damping coefficient, $D$ & 30 \\
Droop, $D_{r}$ & 0.04 \\
Response time, $T_{g}$ & 0.05 \\
\hline
\end{tabular}

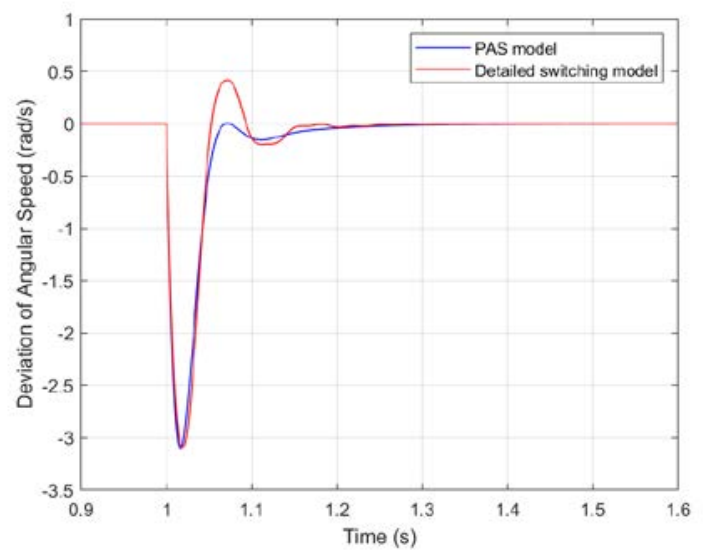

Fig. 6 Verification of the PAS model

\section{Influence of Parameters on Stability}

In this section the influence of parameters $H, D, D_{r}, T_{g}$ on the PAS of the system is investigated. These parameters are investigated separately by varying the investigated parameter in a reasonable range while keeping other parameters' values fixed, equal to the values used in TABLE I. The varying range for each investigated parameter is set by decreasing and increasing certain percentages of the base value listed in TABLE I. The certain percentages are different for each investigated parameter, considering practical limits in reality.

1) Inertia constant $H$

The calculated base value of inertia constant $H$ is 0.1775 . Varying $H$ from 0.1 to 0.5 and plotting the eigenvalue trajectories of the state matrix, the results shown in Fig. 7 are obtained.

From the result we can see there are three eigenvalues, as the system is a third-order system shown in (15). These two conjugated eigenvalues can be referred to as $\lambda_{1}, \lambda_{2}$ and the third one $\lambda_{3}$, same as below. The direction of the arrow

TABLE II. PARAMETERS OF THE DETAILED MODEL

\begin{tabular}{|c|c|c|c|}
\hline Parameter & Value & Parameter & Value \\
\hline Rated voltage, $V_{r e f, l l, r m s}$ & $400 \mathrm{~V}$ & Filter capacitance, $C_{f}$ & $1 \mu \mathrm{F}$ \\
\hline Grid voltage, $V_{g, l l, r m s}$ & $400 \mathrm{~V}$ & Grid inductance, $L_{g}$ & $0.01 \mathrm{H}$ \\
\hline Voltage base, $V_{b}$ & $400 \mathrm{~V}$ & Moment of inertia, $J$ & $0.036 \mathrm{~kg} \cdot \mathrm{m}^{2}$ \\
\hline DC-link voltage, $V_{D C}$ & $800 \mathrm{~V}$ & Damping coefficient, $D$ & $1273.9 \mathrm{~N} \cdot \mathrm{m} \cdot \mathrm{s}$ \\
\hline Rated active power, $P_{r e f}$ & $10 \mathrm{~kW}$ & Droop, $D_{r}$ & 0.04 \\
\hline Rated grid frequency, $f_{n}$ & $50 \mathrm{~Hz}$ & Voltage controller proportional gain, $K_{P v}$ & 0.1 \\
\hline Switching frequency, $f_{s w}$ & $10 \mathrm{kHz}$ & Voltage controller resonant gain, $K_{R v}$ & 500 \\
\hline Sampling frequency, $f_{s}$ & $10 \mathrm{kHz}$ & Current controller proportional gain, $K_{P i}$ & 4.36 \\
\hline Filter inductance, $L_{f}$ & $1.5 \mathrm{mH}$ & & \\
\hline
\end{tabular}


represents the increase of the investigated parameter.

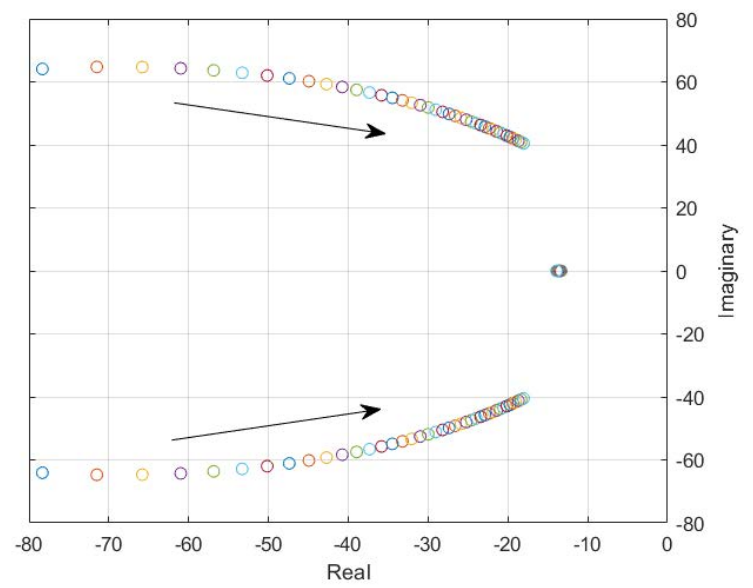

Fig. 7 Eigenvalue trajectories by varying inertia constant $H$

From the figure, it can be found that inertia constant $H$ has little influence on $\lambda_{3}$, while makes $\lambda_{1}, \lambda_{2}$ move towards the imaginary axis. This means the PAS margin is reduced when increasing $H$. This is physically reasonable because a bigger $H$ means the VSM responds slowly to the torque imbalance. This means a longer dynamic process, a longer time to stabilize, and always accompanied by a larger overshoot.

However, it is also intuitive that a larger $H$ is better for the considered grid-forming inverter from the perspective of inertia response, which is also one important aim of utilizing VSM control. A larger $H$ helps reducing the RoCoF and partly improving the nadir of frequency (taking a frequency drop case as an example), which is consistent with the aim of frequency control by implementing VSM control.

Therefore, a compromise needs to be achieved between these two aspects. Moreover, the exact value of $H$ will depend, among others, on the grid code requirements regarding frequency control, while it is also influenced by design choices such as the converter rating. However, the relationship between them is worth being investigated in the future.

\section{2) Damping coefficient $D$}

The calculated base value of the damping coefficient $D$ is 30. Varying $D$ from 10 to 100 and plotting the eigenvalue trajectories of the state matrix, the results shown in Fig. 8 are obtained.

From the figure, it can be seen that there is a range of $D$ that makes the system stable and there is a range of $D$ that makes the system unstable. Easily it can be found out that the system is stable when $D$ is between 10 and 53; the system is unstable when $D$ is between 54 and 100 . These two cases are discussed separately below.

In the stable range, namely $D$ is varying from 10 to 53 , it can be concluded that damping coefficient $D$ has very little influence on $\lambda_{3}$, slightly making it move closer to the imaginary axis by increasing $D$. On the other hand, $D$ has much influence on $\lambda_{1}$ and $\lambda_{2}$, making them move far away from the imaginary axis.

In this range, $\lambda_{3}$ is the dominant eigenvalue as it is closest to the imaginary axis. As it is not changing much in this range and the system is stable, the change of performance of the system mainly is determined by $\lambda_{1}$ and $\lambda_{2}$ when varying the value of $D$. From the result, we can see the performance is improved a lot. This can be explained considering these two conjugated eigenvalues represent periodical oscillating components in the system responses. When these two eigenvalues are moving leftwards and their imaginary parts are getting smaller, it means that the oscillations in the system responses are more quickly damped, in both time and magnitude, which is desirable.

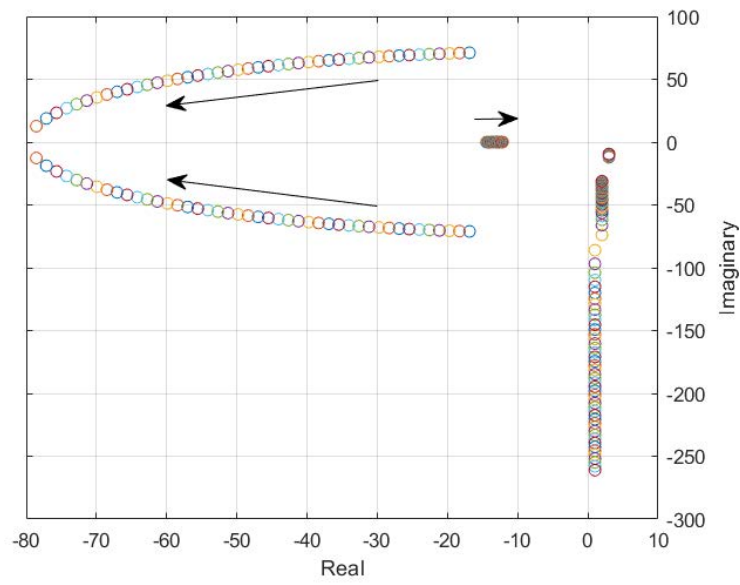

Fig. 8 Eigenvalue trajectories by varying damping coefficient $D$

In the unstable range, the system keeps unstable when increasing $D$ from 54 to 100 . In summary, there is a certain range of $D$ to make the system stable. Therefore, if designing the damping coefficient $D$ only from stability perspective, firstly, we need to find the range of $D$ to keep the system stable; secondly, in this range, its value is supposed to be as large as possible after considering a proper safety margin.

However, the overall design of $D$ also needs to consider the positive effects of a smaller value of $D$. A smaller value means less damped system responses, like larger overshoots. Although larger overshoots are not desirable generally from a stability perspective, a larger overshoot of the active power actually is desirable from the perspective of inertia response and frequency control. It is the immediate and large active power input after the frequency event that enables VSM control to equip a WT with frequency control capability. Therefore, a trade-off needs to be carefully considered between these two aspects.

\section{3) Droop $D_{r}$}

The calculated base value of the droop $D_{r}$ is $4 \%$. Varying $D_{r}$ from 0.01 to 1 and plotting the eigenvalue trajectories of the state matrix, the results shown in Fig. 9 are obtained. 


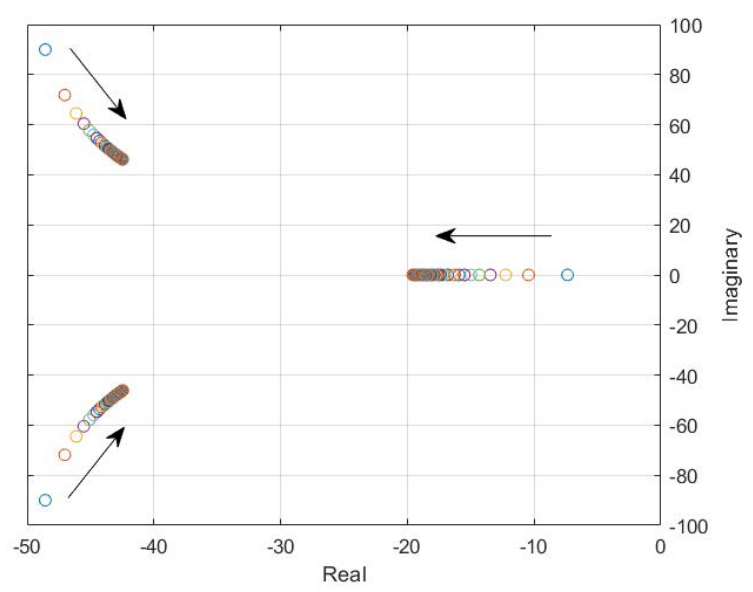

Fig. 9 Eigenvalue trajectories by varying droop $D_{r}$

From the figure, we can see that $D_{r}$ has some influence on $\lambda_{3}$. As the range considered almost covers the entire possible scope of varying the droop, it can be seen that $\lambda_{3}$ is always the dominant eigenvalue in this range. Therefore, the stability of the system is mainly determined by the change of $\lambda_{3}$. That is to say, the stability is improved when the droop is increased. This is physically reasonable because a bigger droop means larger amount of extra power is provided by the VSM to compensate the power imbalance, which is more helpful to maintain the frequency stability by bringing the frequency back to a closer steady state to the rated value.

At the same time considering the change of $\lambda_{1}$ and $\lambda_{2}$, in the process of improving stability by increasing the droop, the magnitude of oscillations of the system response is also reduced. However, the droop cannot be increased as much as we want just to improve the stability, because it is constraint by the capability of the WT to provide or absorb extra power, also it is related with the rating of the inverter and the requirement of grid codes for frequency control capability. Probably stability issue is less important compared with these aspects. Initially and generally, it is reasonable to refer to the practical settings of SGs for the value of the droop, for instance, $4 \%$ [28].

\section{4) Response time}

Here the response time $T_{g}$ represents the time needed to generate or absorb extra power to compensate the power imbalance for frequency control. The base value selected is 0.05 . Varying $T_{g}$ from 0.01 to 10 and plotting the eigenvalue trajectories of the state matrix, the results shown in Fig. 10 are obtained.

From the figure, we can see that the response time also has much influence on $\lambda_{3}$. Summarizing the influence on $\lambda_{3}$ of these four parameters, it is obvious that, basically $\lambda_{3}$ is only influenced by the droop $D_{r}$ and the response time $T_{g}$. This is because this eigenvalue $\lambda_{3}$ is produced by including the governor branch in the control scheme. This can be easily proved by calculating the eigenvalues for the system with a SG only represented by its swing equation. In this case, there are only two conjugated eigenvalues, which are equivalent to $\lambda_{1}$ and $\lambda_{2}$ in this VSM case. Hence, $\lambda_{3}$ is only influenced by the two parameters that are forming the governor branch.

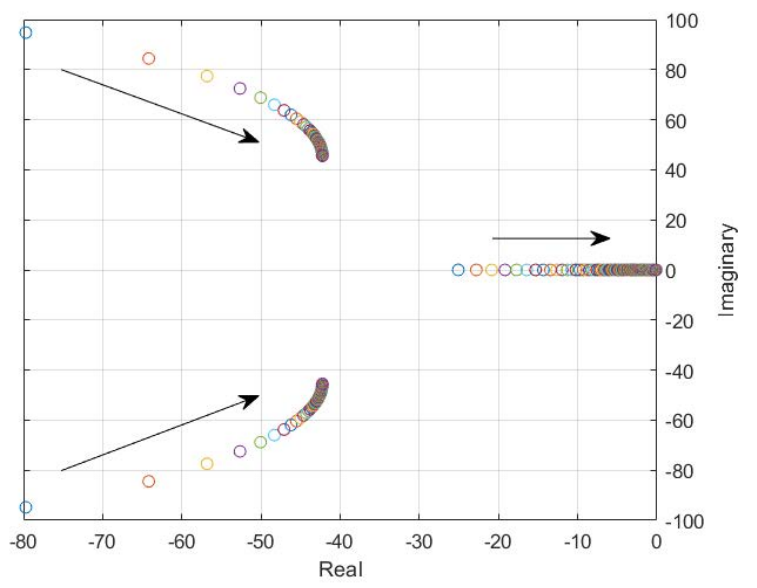

Fig. 10 Eigenvalue trajectories by varying response time $T_{g}$

From Fig. 10, it is easy to conclude that the stability of the system is worsen when increasing $T_{g}$. Therefore, from the perspective of stability, it is better to equip WTs with a quicker responding power source for frequency control capability.

What needs to be noticed from Fig. 10 is that, the system is still stable even when $T_{g}$ is 10 , in which case $\lambda_{3}$ is closest to the origin. Considering that a response time of 10 seconds is already beyond the scope of fast primary frequency control requirement, which is recently proposed by EirGrid, therefore, discussion of $T_{g}$ larger than 10 is beyond the scope of this study.

\section{CONCLUSION}

This paper mainly proposes the concept of power angle small-signal stability (PAS) as a simpler way of analyzing the small-signal stability of the VSM-controlled grid-forming inverters. This simplified method will be of great help in the future for analyzing the rotor angle small-signal stability issue of the power system when there will be large share of converter-interfaced renewable power sources. This method will save much time and computational resources by neglecting the fast dynamics of inner voltage and current control loops of the converter and only considering the power loop, where the variable power angle lies.

Based on this PAS concept and a VSM control scheme proposed earlier, the state-space representation and block diagram are derived for a system, which consists of a VSMcontrolled grid-forming inverter connected to an infinite bus. This PAS model is compared with the original system with minor acceptable errors by time-domain simulations in MATLAB Simulink. Influence of the four main parameters in the power loop on the PAS of the system is investigated in detail. The conclusions are as follows.

Due to the inclusion of the governor branch in the proposed VSM control scheme, a third real eigenvalue is produced for the system, which is only influenced by the two parameters in the governor branch: the droop $D_{r}$ and the response time $T_{g}$.

A larger inertia constant $H$ reduces the stability margin, but enhances the inertia response and frequency control capability of the WT. Hence, a trade-off between both aspects needs to be considered when designing this parameter. 
When designing the damping coefficient $D$, firstly, a range of $D$ needs to be confirmed to guarantee the system remains stable; secondly, a trade-off also needs to be considered between stability margin and frequency control capability.

A larger droop $D_{r}$ helps improving the stability, with its optimal value being more likely constraint by other factors, like rating of extra power source/sink, rating of the inverter and grid code requirements.

A smaller response time $T_{g}$ helps improving the stability.

\section{ACKNOWLEDGMENT}

This work has received funding from the European Union's Horizon 2020 research and innovation program under grant agreement No. 727680 (TotalControl).

This work has also received funding from project PowerKey (EUDP Project No. 12558).

\section{REFERENCES}

[1] D. Turschner and R. Hesse, "Power electronic substitution of a classical synchronous machine for power conditioning in decentralized energy supply," in 3rd French - German conference Renewable and Alternative Energies, 2005, pp. 39-46.

[2] R. Hesse, H. P. Beck, and D. Turschner, "Die virtuelle Synchronmaschine," ETZ Electrical Engineering + Automation, vol. 128, no. S2, pp. 38-44, 2007.

[3] H. P. Beck, R. Hesse, and D. Turschner, "Virtuelle Synchronmaschine in stromrichterdominierten schwachen Netzen," in Zwölftes Kasseler Symposium Energie-Systemtechnik, 2007.

[4] H. P. Beck and R. Hesse, "Virtual synchronous machine," in 2007 9th International Conference on Electrical Power Quality and Utilisation, EPQU, 2007.

[5] L. Lu, Ö. Göksu, and N. A. Cutululis, "A Simplified Virtual Synchronous Machine Control Scheme for Type- 4 Wind Turbines," in 2019 Wind Energy Science Conference, WESC, 2019. Available at: https://orbit.dtu.dk/en/activities/a-virtual-synchronous-machinecontrol-scheme-for-wind-turbines(4cdc26fd-1829-4c00-af625775529c73f3).html

[6] J. Wang, Y. Wang, Y. Gu, W. Li, and X. He, "Synchronous frequency resonance of virtual synchronous generators and damping control," in 9th International Conference on Power Electronics - ECCE Asia: "Green World with Power Electronics", ICPE 2015-ECCE Asia, 2015, pp. 1011-1016.

[7] X. Li, Y. Hu, Y. Shao, and G. Chen, "Mechanism analysis and suppression strategies of power oscillation for virtual synchronous generator," in Proceedings IECON 2017 - 43rd Annual Conference of the IEEE Industrial Electronics Society, 2017, vol. 2017-Janua, pp. 4955-4960.

[8] J. Chen and T. O'Donnell, "Parameter constraints for virtual synchronous generator considering stability," IEEE Trans. Power Syst., vol. 34, no. 3, pp. 2479-2481, 2019.

[9] H. Wu et al., "Small-Signal Modeling and Parameters Design for Virtual Synchronous Generators," IEEE Trans. Ind. Electron., vol. 63, no. 7, 2016.

[10] Z. Wei, C. Jie, and C. Gong, "Small signal modeling and analysis of synchronverters," in 2015 IEEE 2nd International Future Energy Electronics Conference, IFEEC 2015, 2015.

[11] J. Liu, Y. Miura, and T. Ise, "Comparison of Dynamic Characteristics between Virtual Synchronous Generator and Droop Control in Inverter-Based Distributed Generators,” IEEE Trans. Power Electron., vol. 31, no. 5, pp. 3600-3611, 2016.

[12] P. Piya and M. Karimi-Ghartemani, "A stability analysis and efficiency improvement of synchronverter," in Conference Proceedings - IEEE Applied Power Electronics Conference and Exposition - APEC, 2016, vol. 2016-May.

[13] R. Zhang, H. Zhang, W. Feng, and K. Sun, "Small signal analysis of photovoltaic-energy storage integrated virtual synchronous generator," in Proceedings IECON 2017 - 43rd Annual Conference of the IEEE Industrial Electronics Society, 2017, vol. 2017-Janua, pp. 1544-1549.

[14] C. Hu, K. Chen, S. Luo, B. Zhou, and L. Ding, "Small Signal Modeling and Stability Analysis of Virtual Synchronous Generators," in 2017 20th International Conference on Electrical Machines and Systems (ICEMS), 2017, pp. 1-5.

[15] A. Rodriguez-Cabero, J. Roldan-Perez, and M. Prodanovic, "Virtual Impedance Design Considerations for Virtual Synchronous Machines in Weak Grids," IEEE J. Emerg. Sel. Top. Power Electron., 2019.

[16] R. Rosso, J. Cassoli, S. Engelken, G. Buticchi, and M. Liserre, "Analysis and design of LCL filter based synchronverter," in 2017 IEEE Energy Conversion Congress and Exposition, ECCE 2017, 2017, vol. 2017-Janua, pp. 5587-5594.

[17] R. Rosso, S. Engelken, and M. Liserre, "Robust stability analysis of synchronverters operating in parallel," IEEE Trans. Power Electron., 2019.

[18] R. Rosso, J. Cassoli, G. Buticchi, S. Engelken, and M. Liserre, "Robust Stability Analysis of LCL Filter Based Synchronverter Under Different Grid Conditions," IEEE Trans. Power Electron., vol. 34, no. 6, pp. 5842-5853, 2019.

[19] M. Ebrahimi, S. A. Khajehoddin, and M. Karimi-Ghartemani, "An Improved Damping Method for Virtual Synchronous Machines," IEEE Trans. Sustain. Energy, vol. 10, no. 3, pp. 1491-1500, 2019.

[20] S. D'Arco, J. A. Suul, and M. Molinas, "Implementation and analysis of a control scheme for damping of oscillations in VSC-based HVDC grids," in 16th International Power Electronics and Motion Control Conference and Exposition, PEMC 2014, 2014, pp. 586-593.

[21] S. D'Arco, J. A. Suul, and O. B. Fosso, "Small-signal modelling and parametric sensitivity of a Virtual Synchronous Machine," in Proceedings - 2014 Power Systems Computation Conference, PSCC 2014, 2014, pp. 1-9.

[22] S. D’Arco, J. A. Suul, and O. B. Fosso, "Small-signal modeling and parametric sensitivity of a virtual synchronous machine in islanded operation,” Int. J. Electr. Power Energy Syst., vol. 72, pp. 3-15, 2015.

[23] S. D'Arco and J. A. Suul, "Small-Signal analysis of an isolated power system controlled by a virtual synchronous machine," in Proceedings - 2016 IEEE International Power Electronics and Motion Control Conference, PEMC 2016, 2016, pp. 462-469.

[24] O. Mo, S. Darco, and J. A. Suul, "Evaluation of Virtual Synchronous Machines with Dynamic or Quasi-Stationary Machine Models," IEEE Trans. Ind. Electron., vol. 64, no. 7, pp. 5952-5962, 2017.

[25] W. Du, Q. Fu, and H. F. Wang, "Power System Small-Signal Angular Stability Affected by Virtual Synchronous Generators," IEEE Trans. Power Syst., vol. 34, no. 4, pp. 3209-3219, 2019.

[26] C. Sun, G. Joos, and F. Bouffard, "Identification of low-frequency oscillation mode and improved damping design for virtual synchronous machines in microgrid," IET Gener. Transm. Distrib., vol. 13, no. 14, pp. 2993-3001, 2019.

[27] “COMMISSION REGULATION (EU) 2017/1485 of 2 August 2017 establishing a guideline on electricity transmission system operation (Text with EEA relevance),” 2017. [Online]. Available: https://eurlex.europa.eu/legalcontent/EN/TXT/PDF/?uri=CELEX:32017R1485\&from=EN.

[28] P. Kundur, Power System Stability And Control. McGraw-Hill, Inc., 1994.

[29] U. Tamrakar, R. Tonkoski, Z. Ni, and T. M. Hansen, "Current Control Techniques for Applications in Virtual Synchronous Machines," in 2016 IEEE 6th International Conference on Power Systems (ICPS), 2016.

[30] J. Rocabert, A. Luna, F. Blaabjerg, and P. Rodríguez, "Control of power converters in AC microgrids," IEEE Trans. Power Electron., vol. 27, no. 11, pp. 4734-4749, 2012. 